\title{
Calculation of Thermochemical Properties of Carbon-cluster Ablation Species
}

\author{
Maitreyee P. Sharma , \\ University of Illinois at Urbana-Champaign, Urbana,, IL 61801, USA \\ Richard L. Jaffe, ${ }^{\dagger}$ \\ NASA Ames Research Center, Moffett Field, CA, 94035, USA \\ Alessandro Munafò $\ddagger$ Marco Panesi ${ }^{\S}$ \\ University of Illinois at Urbana-Champaign, Urbana, IL, 61801, USA
}

\begin{abstract}
Carbon clusters and hydrocarbons are constituents of the pyrolysis gases injected into the boundary layer of a space vehicle with a carbonaceous heat shield. These molecules have absorption spectra in the VUV and UV region that match the emission spectra of atomic nitrogen and oxygen. Hence, they can potentially absorb the radiation impinging on the heat shield of the space vehicle. This paper studies the ground state thermochemical properties and low-lying excited electronic states of potential radiation absorbing molecules present in the boundary layer using ab initio quantum chemistry methods. These results provide a more accurate prediction of the radiative heat flux on the surface which can lead to improvement in the design of the thermal protection system.
\end{abstract}

\section{Introduction}

During hyperbolic earth entry, returning spacecraft can attain entry speeds of $10-13 \mathrm{~km} / \mathrm{s}$. This results in intense radiation in the shock layer that is directed towards the spacecraft forebody. The radiation is predominantly due to atomic $\mathrm{N}(\mathrm{I})$ and $\mathrm{O}(\mathrm{I})$ lines [1]. One of the main spectral features in the VUV region is the intense $174.29 \mathrm{~nm}$ line of atomic nitrogen. In order to withstand this high radiative heat flux, ablating heat shields are used. Such heat shields have an outer layer which undergoes sublimation and pyrolysis to form a gas layer which carries away some of the heat by convection. During ablation, the pyrolysis gases injected into the boundary layer can absorb a portion of the radiative heat flux coming from the shocked gases. Accounting for this absorption may cause a reduction in the predicted radiative heat flux impinging on the spacecraft and lead to more efficient design of heat shields. Prakash and Park estimated that the reduction in radiative flux could be as large as $12.5 \%$ [2]. Previous studies on $C_{3}$ determined that it is the predominant species injected into the boundary layer by carbonaceous ablating heat shields [3, 4]. $C_{3}$ is known to have a prominent spectral feature in the VUV (centered at $160 \mathrm{~nm}$ [5]), which can absorb the radiative heating due to emission by atomic nitrogen at $174.29 \mathrm{~nm}$ [3]. Strong absorption of VUV radiation at temperatures of $3500-4000 \mathrm{~K}$ which was observed in experiments conducted with shock heated acetylene and methane was attributed to $\mathrm{C}_{3}[2]$ and $\mathrm{C}_{2} \mathrm{H}$ [6]. The spectral range studied was between $140 \mathrm{~nm}$ and 700nm. The $\mathrm{C}_{2} \mathrm{H}$ feature has not been observed. A more recent study by Martin et al. [7] compares various kinetic models used to study the chemistry around a carbon-based phenolic ablative heat shields in air. The boundary layer composition is found using this work. In this study the carbon clusters are limited to

\footnotetext{
*Graduate Research Assistant, Department of Aerospace Engineering, University of Illinois at Urbana-Champaign, 306 Talbot Lab, 104 S. Wright St., Urbana, 61801, IL, USA

${ }^{\dagger}$ Research Physicist, Aerothermodynamics Branch, NASA Ames Research Center, Hampton, CA, 94035, USA, Associate Fellow of AIAA

$\ddagger$ Post Doctoral Research Associate, Department of Aerospace Engineering, University of Illinois at Urbana-Champaign, 306 Talbot Lab, 104 S. Wright St., Urbana, 61801, IL, USA

$\S^{\S}$ Assistant Professor, Department of Aerospace Engineering, University of Illinois at Urbana-Champaign, 306 Talbot Lab, 104 S. Wright St., Urbana, 61801, IL
} 
3 carbon atoms. These previous studies motivate the investigation of spectral properties of $C_{3}$ and other carbon clusters.

The occurrence of carbon clusters in various scientific fields has led to considerable interest in the prediction of the properties of these molecules both experimentally and theoretically. Gingerich et al. [8] determined the formation enthalpies of small linear carbon clusters using high-temperature Knudsen effusion mass spectroscopy. The measured mole fractions of various carbon clusters in the carbon vapor at temperatures between $2500 \mathrm{~K}$ and $3000 \mathrm{~K}$ are used to determine the equilibrium constants, and, ultimately, to back out the formation enthalpies. The earliest efforts to compute the electronic structures of linear carbon clusters were undertaken by Pitzer and Clementi [9, 10]. A 1998 review paper by Van Orden and Saykally [11] surveyed the properties of electronic ground and excited electronic states of carbon clusters, along with the computational methods and experiments that were designed to obtain these values. Since that year, numerous computational studies of the ground states of $\mathrm{Cn}$ and $\mathrm{C}_{n} \mathrm{H}$ species have been published. The most notable for $C_{n}$ species is by Karton et at. [12]. Using state of the art quantum chemistry methods described in the next section, the estimated uncertainties in the formation enthalpies at $0 \mathrm{~K}$ for $\mathrm{C}_{n}(\mathrm{n}=2,10)$ are estimated to be less than $\pm 2 \mathrm{~kJ} / \mathrm{mol}$.

For ablating carbonaceous heat shields, the molecules $C_{n}$ and $C_{n} H(n=1,4)$, are major constituents of the pyrolysis gases. The thermodynamics of $\mathrm{C}_{2}, \mathrm{C}_{3}$ and $\mathrm{C}_{2} \mathrm{H}$ are fairly well known for temperatures up to $10,000 \mathrm{~K}$, but not for other species. For temperatures between 2,000 K and 4,000 K, $\mathrm{C}_{3}$ is known to be the most abundant constituent of carbon vapor in thermochemical equilibrium [8]. Also, the spectroscopy of $\mathrm{C}_{2}$ and $\mathrm{C}_{3}$ has been widely studied. Of particular interest is the strong absorption in the wavelength range 150-180 nm [5], due to the ${ }^{1} \Sigma_{u}^{+} \rightarrow{ }^{1} \Sigma_{g}^{+}$transition of $C_{3}$. Structural, energetic and spectroscopic data for other species are less well known. In this paper we present such data for $\mathrm{C}_{4}, \mathrm{C}_{5}, \mathrm{C}_{3} \mathrm{H}$ and $\mathrm{C}_{4} \mathrm{H}$. Our goal is to determine the abundance of these species in the boundary layers of spacecraft with ablating heat shields during atmospheric entry. In addition, we want to identify other spectroscopic transitions of these species that could results in significant absorption of the radiative heat flux in the shock layer.

The paper is divided into two parts. The first one is the calculation of the ground state and low lying excited state energies and characteristics and the determination of their thermodynamic properties. This is followed by computation of the mole fractions of the molecules in the pyrolysis gases for typical boundary layer conditions, i.e. temperatures between 3,000-7,000 K and pressure of 10,000-25000 Pa for high speed earth reentry vehicles. The second part involves the determination for these specie's excited state electronic energies and transition dipole moments to obtain estimates of the absorption spectral features that could absorb some of the radiative flux due to emission from strong atomic lines of nitrogen and oxygen. Section II gives an overview of the theory and computational methods of quantum mechanics used for the calculation of the properties of various molecules. Subsection A discusses the statistical mechanics theory used in the computation of ground state thermochemical properties using the partition function. The constants for the molecules are computed using quantum chemistry methods which are discussed in this section. Calculation of the low-lying electronic states of the molecules is done by applying time-dependent density functional theory discussed in subsection B Results are presented in section III.

\section{Theoretical and Computation Methods}

In recent years, development of quantum chemistry methods has progressed to the point where molecular thermochemical parameters from accurate computation can equal or surpass experiment in accuracy. In the present study, we use those methods in three different ways as part of a larger effort to characterize the gaseous environment surrounding spacecraft during atmospheric entry. First we compute formation enthalpies for carbon clusters and for hydrocarbon radicals formed in the boundary layer during ablation of the heat shield material. Second, we use this approach to determine the enthalpy, entropy and free energy so that we can compute mole fractions of these species at thermochemical equilibrium. Finally, we survey the electronic excited states of these molecules to identify candidates that could have a role in mitigating the radiative heat flux that impinges on the vehicle. Specifically, quantum chemistry calculations are used to determine potential energy surfaces rotational constants and vibration frequencies that are needed to compute the enthalpy and entropy of the ablation products over the range of temperatures encountered in the boundary layer. We also compute the excitation energies between the ground and excited electronic states and the electronic transition dipole moments between these states in order to identify strong absorption and emission transitions. 
These methods provide approximate, but accurate, solutions of the Schrödinger equation whose analytical solution cannot be found for multi-electron molecules. Since the coupled dynamics of the nuclei and electrons is a complex problem, the Bonn-Oppenheimer approximation is invoked to decouple the electrons and nuclei. The electronic structure is then calculated keeping the nuclei stationary. The standard procedure employed in these methods is approximation of the Hamiltonian to account for all the important contributions to the electronic energy. The following two subsections describe the methods in detail.

\section{A. Ground State Calculations}

To compute the mole fractions of individual species in the boundary layer, one needs to know the enthalpy and entropy of each species for the relevant range of temperature and pressure. These thermodynamic functions are derived from molecular partition functions that are computed from data obtained from state-ofthe-art quantum chemical calculations. The ground state equilibrium geometry and harmonic vibrational frequencies enable approximate calculation of the harmonic oscillator-rigid rotor partition functions. Additional calculations can provide the anharmonic vibration energy levels and rotation-vibration coupling constants, which permit more accurate ro-vibrational partition functions to be determined. The formation enthalpy is determined from a series of accurate calculations for the target species and related molecules. For molecules comprised only of $\mathrm{H}, \mathrm{N}$ and $\mathrm{O}$ atoms, the formation enthalpy can be determined completely from these calculations, by making use of the standard convention that the formation enthalpy of $\mathrm{H}_{2}, \mathrm{~N}_{2}$ and $\mathrm{O}_{2}$ at a pressure of 1 bar and any temperature is zero (i.e. reference state for these elements). When carbon is included, some other reference parameter is needed. In our case, we use the measured formation enthalpy of atomic carbon gas at 1 bar pressure and $0 \mathrm{~K}$. If a particular species has low-lying excited electronic states, the electronic partition function also must be determined for accurate calculation of enthalpy and entropy. Approaches to this are described in section B

Thermodynamic quantities of enthalpy $(\mathrm{H})$, entropy $(\mathrm{S})$ and specific heat $\left(\mathrm{C}_{\mathrm{p}}\right)$ of each species can be expressed in terms of the partition function $Q$. For internal modes (vibration, rotation and electronic excitation),

$$
Q_{\text {int }}=\sum_{i} g_{i} \exp \left(-E_{i} / k_{B} T\right)
$$

where the summation extends over all the energy levels of the particular atomic or molecular species. In the equation, $\mathrm{E}_{i}$ are the energy levels of the species, $\mathrm{T}$ is the temperature, $\mathrm{g}_{i}$ is the degeneracy of the level ' $\mathrm{i}$ ' and $\mathrm{k}_{B}$ is Boltzmanns constant. It is common practice to factor $\mathrm{Q}_{\text {int }}$ into vibration, rotation and electronic components,

$$
Q_{\text {int }}=Q_{\text {rot }} \times Q_{\text {vib }} \times Q_{\text {elec }}
$$

and use the harmonic oscillator-rigid rotor approximation (HO-RR) for $Q_{\text {rot }}$ and $Q_{v i b}$. In this approximation, the same rotation and vibration partition function values are used for each electronic state. If the energy difference between the ground and first electronic states is much larger than $\mathrm{k}_{\mathrm{B}} \mathrm{T}$, where $\mathrm{T}$ is the temperature, this assumption is reasonable. For most molecules rotation-vibration coupling and anharmonic effects are small, making the HO-RR approximation reasonable.

The translation mode partition function $\left(\mathrm{Q}_{\mathrm{tr}}\right)$ is a function of the mass of each species and the temperature.

$$
Q_{t r}=\left(\frac{2 \pi M k_{B} T}{N h^{2}}\right)^{\frac{3}{2}} \frac{R T}{P}
$$

where $\mathrm{M}$ is the molecular or atomic mass of the species, $\mathrm{N}$ is Avogadro's number, $\mathrm{h}$ is Planck's constant, $\mathrm{R}$ is the gas constant and $\mathrm{P}$ is the pressure. The total partition function, $\mathrm{Q}_{T O T}$, is the product of $\mathrm{Q}_{\operatorname{tr}}$ and $\mathrm{Q}_{\text {int }}$.

For modeling gaseous species in the shock and boundary layers, we assume ideal gas behavior and evaluate the thermodynamic functions at the standard state. The standard state specific heat is given by,

$$
C_{p}^{o_{I N T}}=R T^{2} \frac{\partial^{2} \ln Q_{\text {int }}(T)}{\partial^{2} T}+2 R T \frac{\partial \ln Q_{\text {int }}(T)}{\partial T}
$$


The superscript o indicates standard state. The translation contribution to $C_{p}^{o}$ is simply $\frac{5}{2} R$. Therefore, the total $C_{p}$ is,

$$
C_{p}^{o}=C_{p}^{o_{I N T}}+\frac{5}{2} R
$$

For standard state enthalpy,

$$
H^{o}(T)=H^{o}(0)+R T^{2} \frac{\partial \ln Q(T)}{\partial T}
$$

or equivalently,

$$
\frac{H_{i n t}^{o}(T)-H_{i n t}^{o}(0)}{R T}=T \frac{\partial \ln Q_{i n t}(T)}{\partial T}
$$

and,

$$
H_{t r}^{o}(T)-H_{t r}^{o}(0)=\frac{5}{2} R T
$$

$\mathrm{H}^{o}(0)$ must include the formation enthalpy $\left(\Delta_{f} \mathrm{H}^{o}\right)$, which is the difference between the enthalpy of the molecule and its constituent atoms in their standard state. Finally, the standard state entropy is given by,

$$
S^{o}(T)=R \frac{\ln Q(T)}{N}+R T \frac{\partial \ln Q(T)}{\partial T}
$$

It is convenient to use $\mathrm{T}=0 \mathrm{~K}$ as the reference temperature, because only the lowest energy level of each species contributes to the thermodynamic functions.

The $a b$ initio quantum chemistry methods used in this paper for computing formation energies at $\mathrm{T}=$ $0 \mathrm{~K}$ are the so-called hybrid methods [13, 14, 15, 16], particularly G3, G4, CBS-QB3 and the W1RO method. These methods determine the energies of molecules at their equilibrium geometries by combining results obtained with high-level correlation methods (like quadratic configuration interaction theory (QCISD) and coupled cluster theory (CCSD)) using small atomic orbital basis sets with results from lower level methods (like MP2 and MP4) using a large basis set. To increase the accuracy of the final result, an empirical additive correction term is determined as follows. For a large test set of small molecules, a specific property(e.g., atomization energy) is computed using a particular hybrid method and compared with reference values based on experiment. The empirical term is the correction factor that bring the test set results into the best overall agreement with the experimental data. The best hybrid methods G4 and W1ro achieve a mean error of $\pm 2-3 \mathrm{~kJ} / \mathrm{mol}$. This approach is referred to as a composite approach.

The methods mentioned above give the total electronic energy $\left(\epsilon_{0}\right)$, which along with zero-point vibration energy, is used to calculate the atomization energy, $\mathrm{D}_{0}(\mathrm{M})$ at $\mathrm{T}=0 \mathrm{~K}$, using,

$$
D_{0}(M)=\sum x \epsilon_{0}(X)+\sum y \epsilon_{0}(Y)-\epsilon_{0}(M)
$$

where $\mathrm{x}$ and $\mathrm{y}$ stand for number of $\mathrm{X}$ and $\mathrm{Y}$ atoms in molecule $\mathrm{M} . \epsilon_{0}(M)$ include the zero-point energy. The enthalpy of formation $\left(\Delta_{f} \mathrm{H}^{o}\right)$ and Gibbs free energy $\left(\Delta_{f} \mathrm{G}^{\circ}\right)$ of the molecules at $0 \mathrm{~K}$ are computed by,

$$
\begin{gathered}
\Delta_{f} H^{o}(M, 0 K)=x \Delta_{f} H^{o}(X, 0 K)+y \Delta_{f} H^{o}(Y, 0 K)-D_{0}(M) \\
\Delta_{f} G^{o}(M, T)=\Delta_{f} H^{o}(M, T)-T\left[S^{o}(M, T)-\sum_{\text {atoms }} S^{o}(X, T)\right]
\end{gathered}
$$

The equilibrium constant is then calculated in terms of the Gibbs free energy,

$$
K_{\mathrm{eq}}=\exp \left(\frac{\Delta G_{r}^{o}}{k_{B} T}\right)
$$

The $r$ subscript represents the reaction being considered, for example,

$$
a A+b B \rightarrow c C+d D
$$

The equilibrium constant can also be expressed in terms of the partition functions as, 


$$
K_{\mathrm{eq}}=\frac{Q_{C}^{c} Q_{D}^{d}}{Q_{A}^{a} Q_{B}^{b}}
$$

It is important to note that while calculating the equilibrium constant using partition functions, the energies of all species should have a common reference energy.

The software PLATO is used to compute the thermochemical properties and equilibrium compositions. We initially use the mixtures and conditions from reference [6] and [2], to obtain equilibrium mixtures approximating the ones obtained in those studies. The equilibrium calculations are carried for a range of boundary layer temperatures.

\section{B. Electronic Structure Calculations}

It is necessary to characterize the excited states in order to determine their contribution to the enthalpy and entropy and to identify upper states for optical absorption transitions that could take place in the boundary layers. We used two different $a b$ initio quantum chemistry methods, both based on linear-response theory, for calculations of these electronic states. The methods compute the vertical excitation energies of the excited states from the ground electronic state at some particular molecular geometry. By systematically carrying out this calculation for different ground electronic state geometries, the excited state potential energy surfaces can be determined and equilibrium geometries identified for the excited states. The first method used is time dependent density functional theory (TD-DFT) [17]. We used the B3LYP hybrid functional [18] and the augmented correlation-consistent valence triple- $\zeta$ (aug-cc-pVTZ) atomic orbital basis set [19, 20]. The excited electronic states are determined by single electron excitation from the ground state to the higher empty orbitals. The first 30 excited electronic states of molecules are computed using Gaussian 09 package [21]. This provided an effective cut off energy of approximately $8 \mathrm{eV}$, which corresponds to a minimum absorption wavelength $140 \mathrm{~nm}$. The oscillator strength, which determines the strength of the optical transition, is computed between the ground electronic state and all of the excited states. The second method is the equation of motion coupled cluster singles and doubles (EOM-CCSD) [22], also using the the augmented correlation-consistent valence triple- $\zeta$ basis set. This method recovers more of the correlation energy in the excited states and, as a result, gives more accurate excitation energies. These calculations generally have an error of less than $0.1 \mathrm{eV}$ in the excitation energies $(\sim 10 \mathrm{~kJ} / \mathrm{mol})$. For both methods, the ground electronic state geometry and energy are determined at the same level of calculation as are the excitation energies.

\section{Results}

Some molecules of particular interest in this study are $\mathrm{C}_{4}$ (singlet and triplet states), $\mathrm{C}_{5}, \mathrm{C}_{2} \mathrm{H}, \mathrm{CH}_{2}, \mathrm{C}_{3} \mathrm{H}$ and $\mathrm{C}_{4} \mathrm{H}$ (linear and cyclic molecule) which are hypothesized to have absorption characteristics in VUV and UV spectral ranges. For $C_{n}$ molecules, even ' $n$ ' molecules have low lying singlet and triplet states which are nearly isoenergetic whereas odd ' $n$ ' molecules have low lying linear singlet states and higher energy triplets. For $\mathrm{C}_{4}$, the linear triplet structure is the ground state $\left({ }^{3} \Sigma_{g}^{-}\right)$, but the cyclic singlet state $\left({ }^{1} A_{g}\right)$ is close in energy. In contrast, $\mathrm{C}_{5}$ has a close shell $\left({ }^{1} \Sigma_{g}^{+}\right)$ground state with a linear structure. $\mathrm{C}_{4} \mathrm{H}$ also has two isomers, linear and cyclic, that have energies close to each other. The objective of this paper is to use accurate quantum chemistry methods to study these molecules and ascertain if any of them have radiation blocking capabilities.

Before computing the thermochemical properties and spectral features of the test molecules, a validation study is done to compare the results with those available in the literature. $C_{3}$ is treated as an example molecule and is used to validate the approach adopted in this paper. Further in this section molecules with favourable spectral characteristics are recognized and equilibrium mole fractions of these species at the conditions of the boundary layer are estimated using the thermochemical library, PLATO. This estimation is necessary to understand the potential impact these carbon clusters can have on the radiation due to state transitions in atomic species found in the shock layer. 


\section{A. Computation and Validation of atomization energy at $0 \mathrm{~K}$}

The first validation study consists of comparing the atomization energy of the lowest singlet and triplet states of $C_{2}$ and $C_{4}$ molecules and lowest singlet state of $C_{3}$ and $C_{5}$ to the results from Karton et al. [12]. The following table shows the comparison for different methods used in Gaussian to compute the thermochemical properties. It is observed that the results have good agreement, within $\pm 1 \mathrm{kcal} \mathrm{mol}^{-1}$. The methods used for these calculations have been discussed earlier in section IA.

Table 1: Comparison of total atomization energies at 0K with Karton et al. [12] (in kcal mol ${ }^{-1}$ )

\begin{tabular}{ccccc}
\hline Molecule & CBS-QB3 & G4 & W1ro & Best Est. [12] \\
\hline$C_{2}\left({ }^{1} \Sigma_{g}^{+}\right)$ & 144.71 & 147.46 & 143.59 & 144.07 \\
\hline$C_{2}\left({ }^{3} \Pi_{g}\right)$ & 140.25 & 142.47 & 145.90 & 142.39 \\
\hline$C_{3}\left({ }^{1} \Sigma_{g}^{+}\right)$ & 314.26 & 317.03 & 315.36 & 315.83 \\
\hline$C_{4}\left({ }^{3} \Sigma_{g}^{-}\right)$ & 426.86 & 428.87 & 431.07 & 429.16 \\
\hline$C_{4}\left({ }^{1} A_{g}\right)$ & 427.56 & 431.82 & 429.97 & 430.09 \\
\hline$C_{5}\left({ }^{1} \Sigma_{g}^{+}\right)$ & 594.50 & 598.45 & 596.67 & 596.64 \\
\hline
\end{tabular}

\section{B. Validation Study with Existing Thermochemical Tables}

Active Thermochemical Tables (ATcT) [23, 24, 25] is a database of thermochemical properties for molecules developed at the Argonne National Laboratory. The properties are calculated by taking a weighted average of values from literature, which include experimental and computational results. A validation study of the formation enthalpy computed using Gaussian is done by comparison with the ATcT results. The thermochemical data is also compared to JANAF Tables [26] and Gurvich Thermo Tables[27] whose values are widely used for the prediction of mixture composition in the boundary layer of an ablating heat shield.

The test molecules formation enthalpies have good agreement with the ATcT, with differences mostly in a range of $\pm 3 \mathrm{kcal} \mathrm{mol}^{-1}$. Thermochemical properties for molecules like $\mathrm{C}_{3} \mathrm{H}$ and $\mathrm{C}_{4} \mathrm{H}$ which are not available in the previously mentioned tables have been determined in this paper. Table 3 catalogs a few such molecules studied in this paper.

Table 2: Comparison of $\Delta_{f} H$ at $0 \mathrm{~K}$ with Existing Thermochemical Tables (in $\mathrm{kcal} \mathrm{mol}^{-1}$ )

\begin{tabular}{cccccc}
\hline Molecule & CBS-QB3 & G4 & ATcT & JANAF [26] & Gurvich [27] \\
\hline $\mathrm{C}_{2}\left({ }^{1} \Sigma_{g}^{+}\right)$ & 195.25 & 192.50 & $196.04 \pm 0.06$ & 198.19 & 196.56 \\
$\mathrm{C}_{3}\left({ }^{1} \Sigma_{g}^{+}\right)$ & 195.68 & 192.91 & $194.68 \pm 0.13$ & 193.94 & 198.61 \\
$\mathrm{C}_{3}\left({ }^{3} \Pi_{u}\right)$ & 216.96 & 216.01 & $214.91 \pm 0.36$ & - & - \\
$\mathrm{C}_{4}\left({ }^{1} A_{g}\right)$ & 252.36 & 248.10 & 250.34 & 230.43 & 244.98 \\
$\mathrm{C}_{2} \mathrm{H}\left({ }^{2} \Sigma^{+}\right)$ & 135.86 & 133.84 & $134.77 \pm 0.04$ & 113.27 & 135.04
\end{tabular}




\begin{tabular}{cccccc}
$\mathrm{CH}\left({ }^{2} \Pi\right)$ & 141.58 & 140.50 & $141.68 \pm 0.03$ & 141.17 & 141.98 \\
$\mathrm{C}_{2} \mathrm{H}_{2}\left({ }^{1} \Sigma_{g}^{+}\right)$ & 56.02 & 54.76 & $54.69 \pm 0.03$ & 54.32 & 54.48 \\
$\mathrm{CH}_{4}\left({ }^{1} \Sigma_{g}^{+}\right)$ & -15.95 & -15.93 & $-15.91 \pm 0.01$ & -15.99 & -15.92 \\
\hline
\end{tabular}

Table 3: Determination of $\Delta_{f} H$ at $0 \mathrm{~K}$

\begin{tabular}{cccccc}
\hline Molecule & CBS-QB3 & G4 & ATcT & JANAF & Gurvich \\
\hline $\mathrm{C}_{3} \mathrm{H}\left({ }^{2} \Pi\right)$ & 171.39 & 169.01 & - & - & - \\
$\mathrm{C}_{3} \mathrm{H}$ (Linear) & 175.66 & 170.66 & - & - & - \\
$\mathrm{C}_{4} \mathrm{H}\left({ }^{2} \Sigma_{g}^{+}\right)$ & 191.68 & 190.54 & - & - & - \\
$\mathrm{C}_{4} \mathrm{H}($ Cyclic $)$ & 215.29 & 212.023 & - & - & - \\
\hline
\end{tabular}

\section{C. $\mathrm{C}_{3}$ - Example and Validation}

$\mathrm{C}_{3}$ (shown in Figure 1a) has been studied previously by Jaffe et al. [3] and Schwenke [28]. They identified a strong transition from the ground state to the first ${ }^{1} \Sigma_{u}^{+}$electronic state that can be effective in adsorption of the radiation flux for the wavelength range 160-190 nm. Figure $1 \mathrm{~b}$ represents the bending potential for the $C_{3}$ molecule obtained using TD-DFT method with augmented correlation-consistent valence triple- $\zeta$ (aug-cc-pVTZ) basis set. The diamond markers in the figure show the MRCI results from Ref. [3]. This comparison shows that the results are in good agreement for some of the states. However, a few states are missing when using the TD-DFT method to obtain the excited electronic state. Hence, for future calculations, the Molpro quantum chemistry package[29] will be used to obtain more accurate energies of the excited electronic states. The ${ }^{1} \Sigma_{u}^{+}$state is state number 8 in the figure. Note that it has a minimum potential at $\phi=120^{\circ}$.

Figure 2 shows measured and simulated spectra for the VUV absorption feature in $C_{3}$. The experimental spectra [5] were obtained for $\mathrm{C}_{3}$ in cryogenic noble gas matrices $(4.3 \mathrm{~K}$ in Ne and $10 \mathrm{~K}$ in Ar). These are the only published spectra for this absorption transition. The simulated spectra were determined by D. W. Schwenke [28] and are based on accurate quantum chemical calculations similar to those described in Ref. [3]. The measured and simulated absorbance of $\mathrm{C}_{3}$ at $4.3 \mathrm{~K}$ are compared in Figure 2a and the simulated spectrum at $3000 \mathrm{~K}$ is shown in Figure $2 \mathrm{~b}$. Note that the peak absorbance at $4.3 \mathrm{~K}$ occurs around $155 \mathrm{~nm}$, while at $3000 \mathrm{~K}$ it occurs around $174.29 \mathrm{~nm}$. The high temperature spectrum also does not exhibit the band structure seen at lower temperatures. Therefore, it is necessary to run more extensive calculations to obtain accurate results for the temperature range encountered in the boundary layer. 


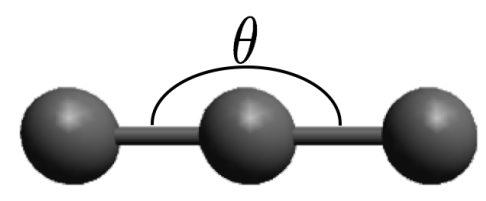

(a) $\mathrm{C}_{3}$ Molecule Ground State

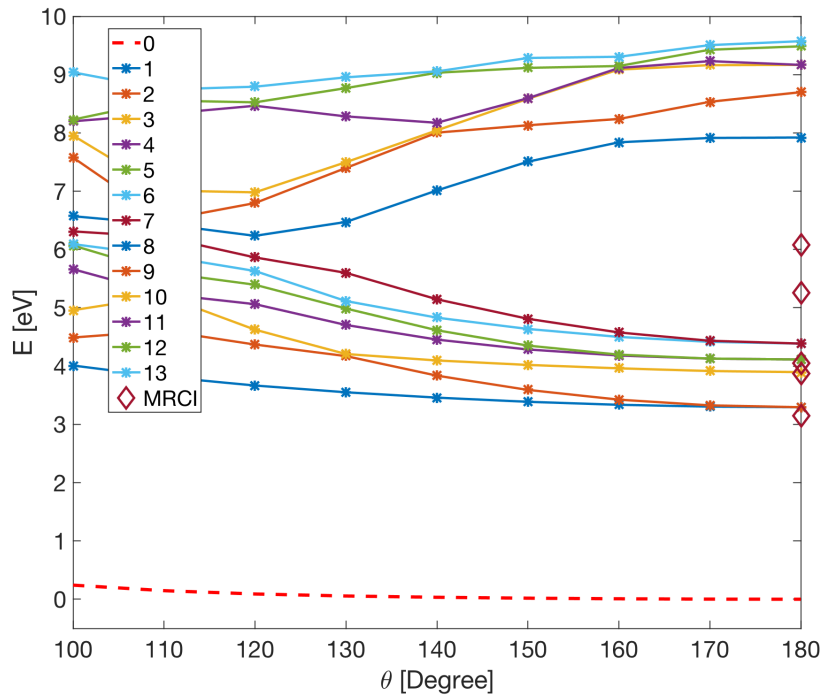

(b) Bending potential from TD-DFT calculations for the lower electronic states of $\mathrm{C}_{3}$. MRCI results from Reference [3]

Figure 1: Comparison of results of quantum chemistry calculations for $\mathrm{C}_{3}$

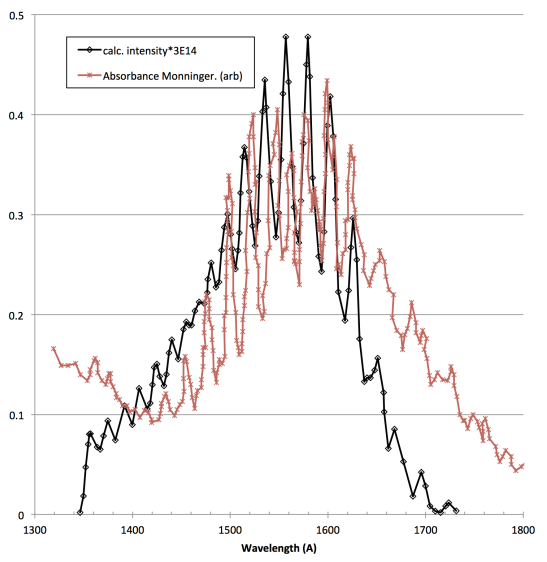

(a) Absorption Spectrum at $4.3 \mathrm{~K}$

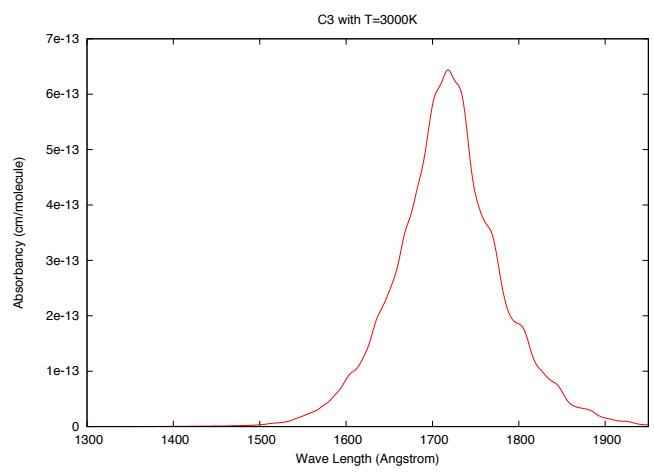

(b) Absorption Spectrum at $3000 \mathrm{~K}$

Figure 2: Absorption Spectra of $\mathrm{C}_{3}$ at different temperatures. Black line is the experimental spectrum (Monninger et al. [5]), and red line is simulated spectrum (Schwenke [28]) 


\section{Nitrogen Atom Transitions}

Table 4 lists the favourable electronic transitions of the test molecules which have an oscillator strength greater than 0.1. The linear isomer of $\mathrm{C}_{4} \mathrm{H}$ has a strong spectral feature close to the $174.29 \mathrm{~nm}$ line of atomic nitrogen. This molecule has a doublet ground state. $\mathrm{C}_{4}$ triplet state also has a very strong absorption line corresponding to the wavelength of $176.97 \mathrm{~nm}$. This is the first indication that these molecules may provide significant radiation blockage in the boundary layer.

A similar comparison was done for the lines of $\mathrm{O}(\mathrm{I})$. However, it is observed that there are no good candidates for absorption of radiation from atomic oxygen.

Table 4: Excitation energies and oscillator strengths matching atomic nitrogen spectra:

\begin{tabular}{clccc}
\hline $\begin{array}{c}\text { Spectral } \\
\text { Line of N [nm] }\end{array}$ & Molecules Studied & $\begin{array}{c}\text { Energy } \\
{[\mathrm{eV}]}\end{array}$ & $\begin{array}{c}\text { Energy } \\
{[\mathrm{nm}]}\end{array}$ & $\begin{array}{c}\text { Oscillator } \\
\text { Strength (f) }\end{array}$ \\
\hline 119.9550 & $\mathrm{C}_{2}$ Excited State 18: & 10.4391 & 118.77 & 0.1656 \\
124.3179 & $\mathrm{C}_{2}$ Excited State 15: & 9.8960 & 125.29 & 0.4531 \\
174.2729 & $\mathrm{C}_{4}$ Excited State 23: & 6.7413 & 183.92 & 1.4173 \\
174.2729 & $\mathrm{C}_{4}$ H Linear Excited State 19: & 7.0061 & 176.97 & 0.9360 \\
174.2729 & $\mathrm{C}_{4}$ H Linear Excited State 22: & 7.1961 & 172.29 & 0.1215 \\
\hline
\end{tabular}

\section{E. $\mathrm{C}_{2}$ Singlet and Triplet State}

The $\mathrm{C}_{2}$ molecule is a two carbon atom molecule with a $\mathrm{C}-\mathrm{C}$ triple bond. Like other even numbered carbon clusters, $C_{2}$ has close low energy singlet and triplet states, with the triplet state $\left({ }^{3} \Pi_{u}\right)$ having an energy 0.088 $\mathrm{eV}$ higher than the ground singlet state $\left({ }^{1} \Sigma_{g}^{+}\right)$. The ground electronic state has its minimum at $r=1.2511 \AA$. The $\mathrm{C}_{2}$ molecule has strong absorption in the lower wavelengths region. The transition corresponding to $125.29 \mathrm{~nm}\left({ }^{1} \Sigma_{g}^{+} \leftarrow^{1} \Sigma_{u}^{+}\right)$has a high oscillator strength. This excited electronic state is marked as state 15 in figure $3 \mathrm{a}$. The other state having a promising absorption feature corresponds to state 18 in the figure. The energy difference between the ground state $\left({ }^{1} \Sigma_{g}^{+}\right)$and this excited state $\left({ }^{1} \Pi_{u}\right)$ is $118.77 \mathrm{~nm}$. Later sections show that the mole fraction of $C_{2}$ at the conditions prevailing in the boundary layer are non-negligible.

Table 5: Electronic States of $C_{2}$ Molecule

\begin{tabular}{ccc}
\hline Energy $[\mathrm{eV}]$ & Degeneracy & Term Symbol \\
\hline 0.0 & 1 & ${ }^{1} \Sigma_{g}^{+}$ \\
0.0888 & 6 & ${ }^{3} \Pi_{u}$ \\
0.7978 & 3 & ${ }^{3} \Sigma_{g}^{-}$ \\
1.0404 & 2 & ${ }^{1} \Pi_{u}$ \\
1.1441 & 3 & ${ }^{3} \Sigma_{u}^{+}$ \\
\hline
\end{tabular}




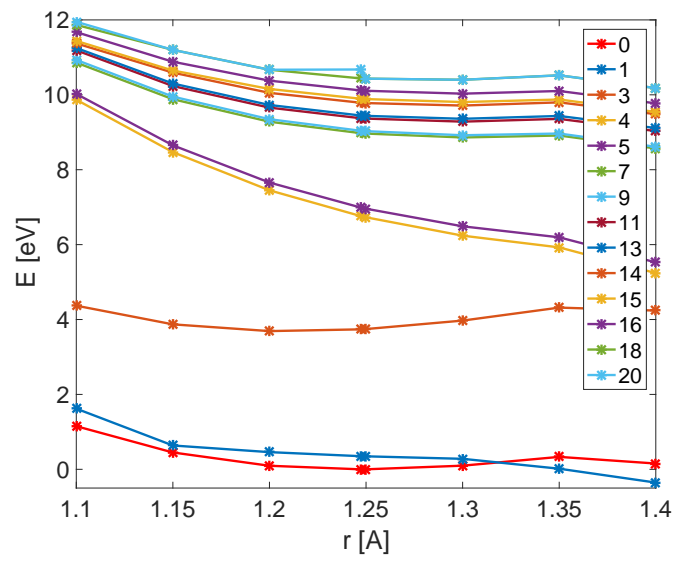

(a)

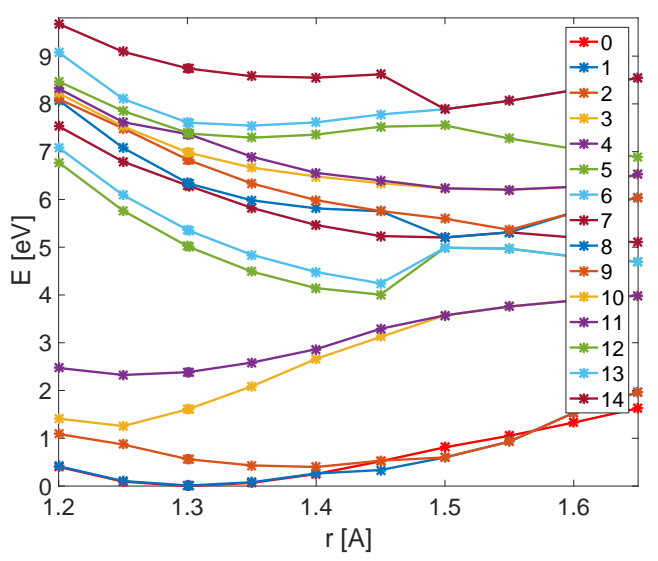

(b)

Figure 3: Potential from TD-DFT calculations for the lower electronic states of $C_{4}$

\section{F. $\mathrm{C}_{4}$ and $\mathrm{C}_{4} \mathrm{H}$ Linear Isomers}

The $\mathrm{C}_{4}$ molecule is a carbon cluster chain with low energy singlet and triplet states. The singlet state has a rhombus geometry and has a lower formation enthalpy of $1047.41 \mathrm{~kJ} / \mathrm{mol}$ at $0 \mathrm{~K}$. The triplet state is a linear isomer having a formation enthalpy of $1052.36 \mathrm{~kJ} / \mathrm{mol}$ at $0 \mathrm{~K}$. The triplet ground state energy is 0.706 $\mathrm{eV}$ lower than the singlet ground state. As observed in the previous section, the $\mathrm{C}_{4}$ triplet state has a very strong absorption line corresponding to the wavelength of $176.97 \mathrm{~nm}$. This absorption is due to the transition between the ${ }^{3} \Sigma_{g}^{-}$and ${ }^{3} \Sigma_{u}^{-}$state. The optimum bond length which minimizes the potential of the ground state corresponds to a bond length of $1.305 \AA$ for the outer atoms and $1.2863 \AA$ bond length of the middle two carbon atoms. The minimum of the ${ }^{3} \Pi$ excited electronic state is at a bond length of around $1.4 \AA$ for the outer atoms.

Table 6 lists the first few electronic states of the $\mathrm{C}_{4}$ molecule. Figure 4 shows the potential energy curves obtained by varying the bond lengths ' $r$ ' of the outer carbon atoms as shown in figure 4a The lowest red curve represents the ground ${ }^{3} \Sigma_{g}^{-}$state with a minima at $r=1.305 \AA$. The excited electronic states are obtained by using methods discussed in Section II B

Table 6: Electronic States of $\mathrm{C}_{4}$ Molecule

\begin{tabular}{ccc}
\hline Energy $[\mathrm{eV}]$ & Degeneracy & Term Symbol \\
\hline 0 & 3 & ${ }^{3} \Sigma_{g}$ \\
0.743905185 & 6 & ${ }^{3} \Pi_{g}$ \\
2.425626839 & 3 & ${ }^{3} \Sigma_{u}^{+}$ \\
2.975620739 & 6 & ${ }^{3} \Delta$
\end{tabular}




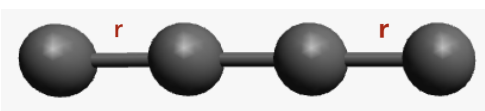

(a) Ground state geometry

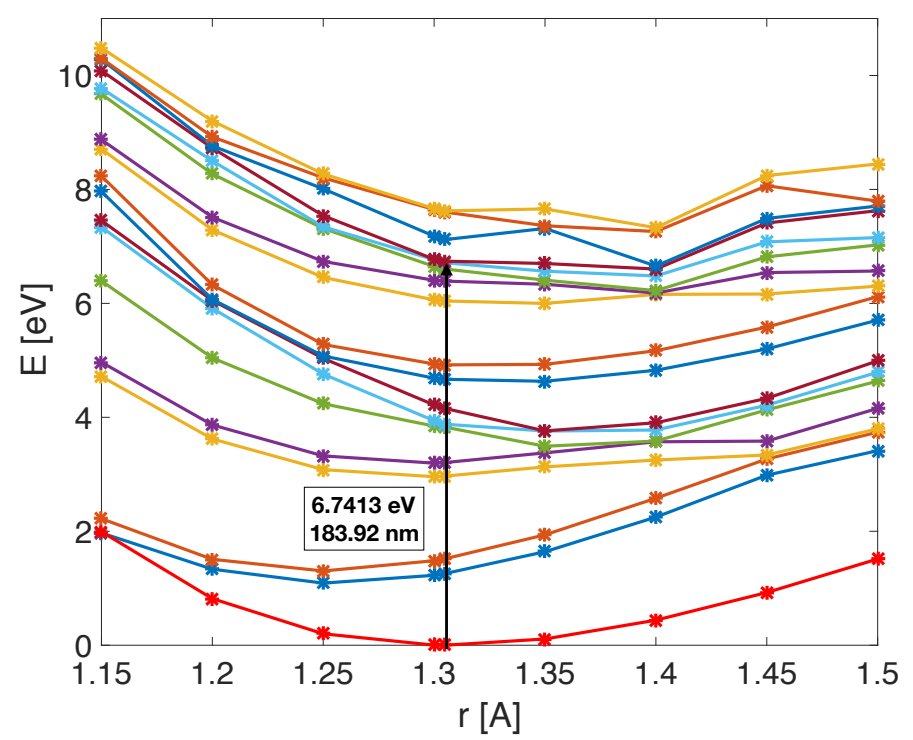

(b) Potential from TD-DFT calculations for the lower electronic states of $C_{4}$

Figure 4: Results of quantum chemistry calculations for $\mathrm{C}_{4}$

Another molecule of interest is the linear isomer of $\mathrm{C}_{4} \mathrm{H}$ molecule. The minimum potential of the ground electronic state occurs at an angle of $180^{\circ}$ of the $\mathrm{C}-\mathrm{H}$ bond. The potential energy curves in this case are obtained for a varying $\mathrm{C}-\mathrm{H}$ bond angle ' $\theta$ '. It is observed from figure $5 \mathrm{~b}$ that symmetry breakdown occurs as the bond angle is lowered, lowering the symmetry group of the molecule. The transition of interest in this molecule from the doublet ground state, ${ }^{2} \Sigma^{+}$, to the excited state having an energy of $7.0061 \mathrm{eV}$ above the ground state, ${ }^{2} \Sigma^{+}$. The current work uses unrestricted Hartree Fock method and DFT to computed the molecular orbitals. However, these methods are unable to describe the degenerate states and hence the electronic levels need to be further analysed and computed using more accurate methods like CCSD(Coupled Cluster Single Double) theory.

Table 7: Electronic States of $\mathrm{C}_{4} \mathrm{H}$ Molecule

\begin{tabular}{ccc}
\hline Energy $[\mathrm{eV}]$ & Degeneracy & Term Symbol \\
\hline 0.0 & 2 & ${ }^{2} \Sigma^{+}$ \\
0.5045 & 2 & ${ }^{2} \Sigma^{-}$ \\
3.3008 & 6 & ${ }^{2} \Pi$ \\
3.4044 & 4 & ${ }^{4} \Sigma$ \\
3.8586 & 6 & ${ }^{2} \Pi$ \\
4.2189 & 12 & ${ }^{4} \Pi$ \\
& & \\
\hline
\end{tabular}




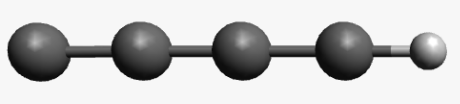

(a) Ground state geometry

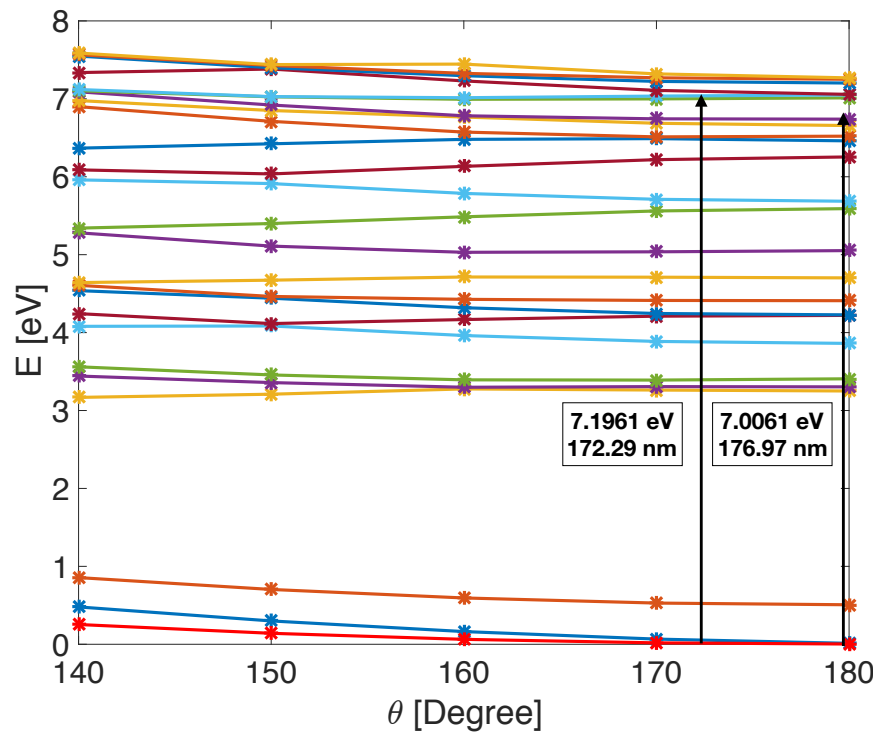

(b) Bending potential from TD-DFT calculations for the lower electronic states of $\mathrm{C}_{4} \mathrm{H}$

Figure 5: Results of quantum chemistry calculations for $\mathrm{C}_{4} \mathrm{H}$

\section{G. Equilibrium Compositions in the Boundary Layer}

After the observations made in the previous subsection, it becomes necessary to estimate the mole fractions of these species in the boundary layer in order to qualitatively estimate their effect on the radiative heat flux impinged on the surface of the vehicle. To compute the equilibrium composition, initial mixture conditions are obtained from shock tube experiments conducted in the 1980s, [6] and [2]. The first case is a mixture of $99 \%$ Argon and $1 \% \mathrm{C}_{2} \mathrm{H}_{2}$ at an initial pressure of $0.76 \mathrm{kPa}$. Post shock pressure for this case is calculated using PLATO. The second mixture is composed of $98 \%$ Argon and $2 \% \mathrm{CH}_{4}$ at an initial pressure of $0.62 \mathrm{kPa}$. Fig 6 shows the evolution of the equilibrium mole fractions of the species. A temperature range from 1000 $\mathrm{K}$ to $7000 \mathrm{~K}$ is considered. Typical temperatures in the boundary layer are in a range of $3000 \mathrm{~K}$ to $7000 \mathrm{~K}$.

The species that are of interest are the $\mathrm{C}_{4}$ Triplet state and the $\mathrm{C}_{4} \mathrm{H}$ doublet linear state. When starting with a mixture of $99 \%$ Argon and $1 \% \mathrm{C}_{2} \mathrm{H}_{2}$, the mole fractions of $\mathrm{C}_{4}$ triplet, indicated as $\mathrm{C}_{4}-\mathrm{T}$, and $\mathrm{C}_{4} \mathrm{H}$ are below $10^{-8}$ for all temperatures. Using $2 \% \mathrm{CH}_{4}$ in Argon at a pressure of $15000 \mathrm{~Pa}$, the mole fraction of $\mathrm{C}_{4}$ - $\mathrm{T}$ is still low. However, at lower temperatures, we observe high mole fractions of $\mathrm{C}_{4} \mathrm{H}$. Presence of these species in significant amount in the boundary layer will lead to the absorption of a large amount of radiation from the shock layer.

Equilibrium mole fractions are also studied for a pure carbon vapor at a pressure of $15000 \mathrm{~Pa}$. At lower temperatures, $\mathrm{C}_{3}$ is the most stable carbon cluster and hence is the most abundant molecule. We also notice the presence of $C_{4}$ triplet at low temperature with the mole fractions on the order of $10^{-8}$.

To study the evolution of mole fractions as a function of pressure, equilibrium calculations for pure acetylene and methane mixtures are carried out at $3000 \mathrm{~K}$ with pressures varying from 10,000-25,000 $\mathrm{Pa}$. The mole fractions are less sensitive to the pressure variation when compared to their temperature dependence. Figure 7 depicts this behavior. 


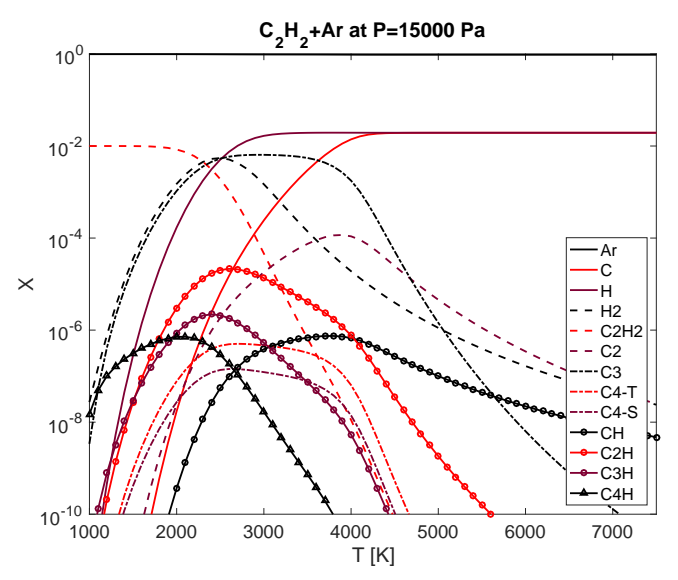

(a) $1 \% \mathrm{C}_{2} \mathrm{H}_{2}+99 \% \mathrm{Ar}$

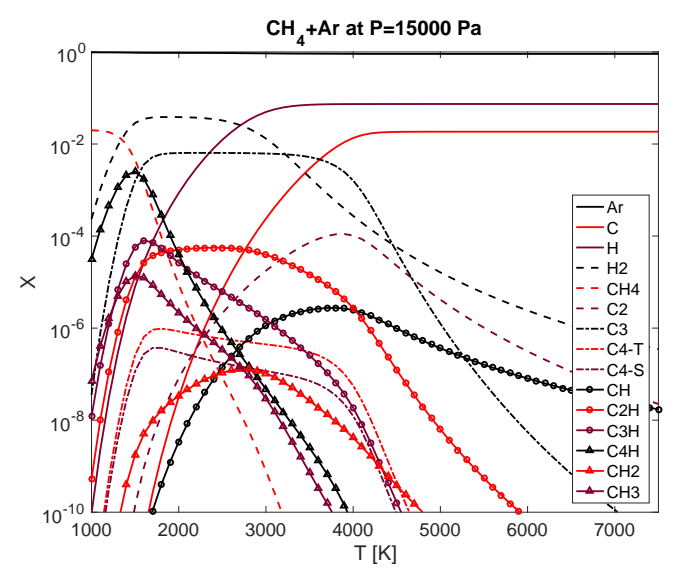

(b) $2 \% \mathrm{CH}_{4}+98 \% \mathrm{Ar}$

Figure 6: Equilibrium Mole fractions at $\mathrm{P}=15,000 \mathrm{~Pa}$

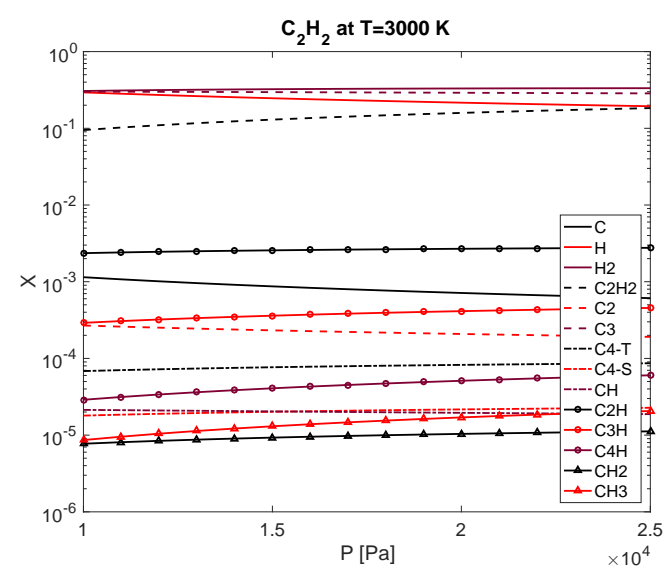

(a) $100 \% \mathrm{C}_{2} \mathrm{H}_{2}$

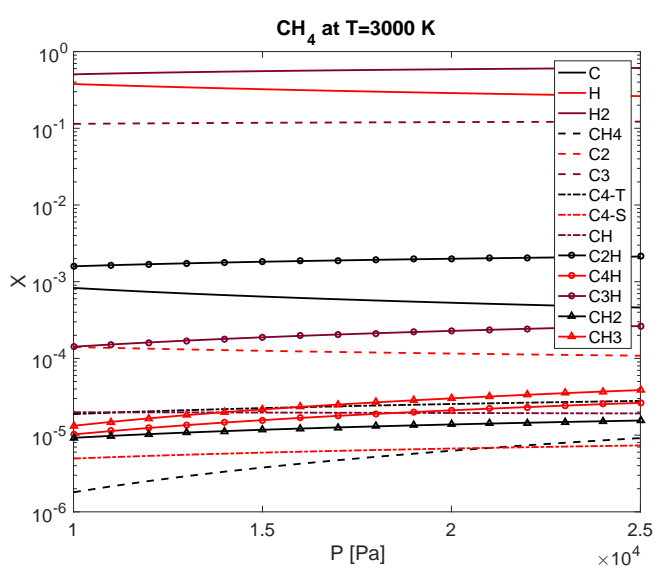

(b) $100 \% \mathrm{CH}_{4}$

Figure 7: Equilibrium Mole fractions at $\mathrm{T}=3000 \mathrm{~K}$

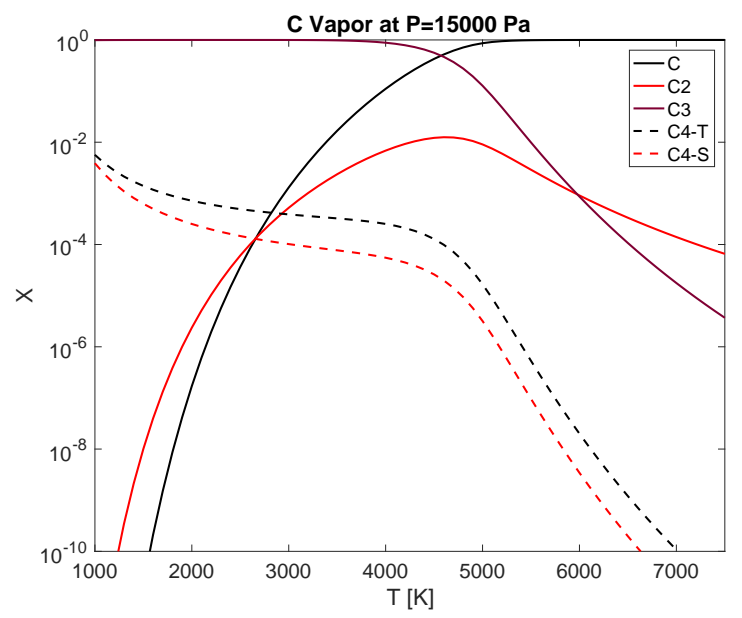

Figure 8: Equilibrium Mole fractions with $100 \% \mathrm{C}$ vapor at 15,000 Pa 


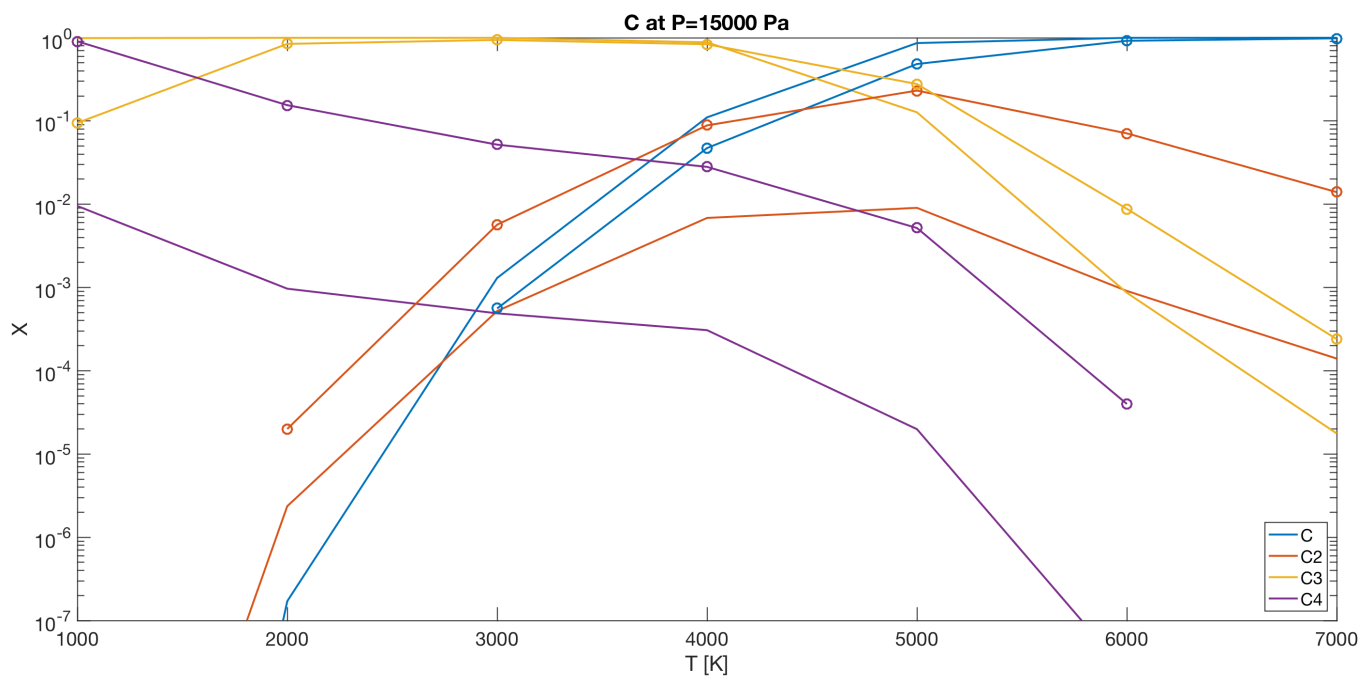

Figure 9: Comparison Equilibrium Mole fractions of pure $C$ vapor between CEA(solid line symbols) and this work(solid line) at $15,000 \mathrm{~Pa}$

In this study, we aim to provide more accurate thermochemical data for the favorable molecules, i.e. molecules that can lead to significant absorption of radiation. Hence, as a comparison, the equilibrium mole fractions for pure carbon vapor at 15,000 $\mathrm{Pa}$ are compared with those obtained from CEA [30]. There is a significant difference in the mole fractions of the $C_{2}$ and $C_{4}$ molecule. Therefore, for more accurate thermochemistry, it is required that these molecules be studied further in detail which is what we aim to achieve through this work.

The equilibrium calculations show that these species have non-negligible mole fractions in the equilibrium mixtures at conditions similar to the ones occurring in the boundary layer. The presence of these species implies that there can be a significant decrease in the radiative heat flux on the surface of the spacecraft. Hence, these molecules should to be studied in further detail and be considered during the calculation of heat flux on the surface of a re-entry vehicle.

\section{Conclusion}

This paper proposes the study of carbon clusters appearing as products of pyrolysis during ablation. The paper aims to quantitatively estimate their presence in the boundary layer around an ablative heat shield and qualitatively study their effect on the radiative heat flux reaching the surface of the vehicle. The approach is divided into two sections, the first is to recognize molecules with favorable absorption spectra when compared to the emission spectra of atomic nitrogen and oxygen and the second is estimating equilibrium mole fractions of these favorable species. Time dependent density functional theory is used to obtain the low lying electronic energy states of the molecules. Gaussian software is used to run these calculations. The emission spectra $\mathrm{N}(\mathrm{I})$ and $\mathrm{O}(\mathrm{I})$ are obtained from the NIST database. The ground state thermochemical properties are calculated using methods like CBS-QB3 and G4MP2 from the Gaussian software. Once the thermochemical properties are calculated, equilibrium mole fractions are calculated using PLATO, a thermochemical library.

The electronic state calculations reveal that $\mathrm{C}_{4}$ triplet ground state and $\mathrm{C}_{4} \mathrm{H}$ linear isomer have strong transitions corresponding to the $174.29 \mathrm{~nm}$ line of $\mathrm{N}(\mathrm{I})$. This is the first indication of possible radiation blockage from these molecules.

The equilibrium mole fractions of these relevant species are observed to be significant and could effect the radiative heat flux. Comparisons with equilibrium mole fractions from widely used codes like CEA, show considerable differences and hence this calls for a need to study the appropriate molecules in further detail in order to obtain accurate thermochemistry data. In future, we aim to study the molecules with favorable spectral features using more accurate $a b$ initio calculations for excited electronic states using Molpro quantum chemistry package [29]. 


\section{Acknowledgments}

This work was supported by Analytical Mechanics Associates Inc. Summer Internship Program at NASA Ames Research Center.

\section{References}

1 AM Brandis, CO Johnston, BA Cruden, and DK Prabhu. Equilibrium radiative heating from 9.5 to 15.5 km/s for earth atmospheric entry. Journal of Thermophysics and Heat Transfer, 31(1):178-192, 2016.

2 S.G. Prakash and C. Park. Shock tube spectroscopy of $\mathrm{C}_{3}+\mathrm{C}_{2} \mathrm{H}$ mixture in the 140-700 nm range. AIAA Paper, 94:1979, 1979.

${ }^{3}$ R. L. Jaffe, G. Chaban, and D. Schwenke. Theoretical determination of high-temperature absorption spectra for $\mathrm{C}_{3}$ in the near-UV and VUV. In $43^{\text {rd }}$ AIAA Thermophysics Conference, page 2743.

${ }^{4}$ C. Park, R. L. Jaffe, and H. Partridge. Chemical-kinetic parameters of hyperbolic earth entry. Journal of Thermophysics and Heat transfer, 15(1):76-90, 2001.

${ }^{5}$ G. Monninger, M. Förderer, P. Gürtler, S. Kalhofer, S. Petersen, L. Nemes, P. G. Szalay, and W. Krätschmer. Vacuum ultraviolet spectroscopy of the carbon molecule $\mathrm{C}_{3}$ in matrix isolated state: experiment and theory. The Journal of Physical Chemistry A, 106(24):5779-5788, 2002.

6 J. L. Shinn. Optical absorption of carbon and hydrocarbon species from shock-heated acetylene and methane in the 135-220 nm wavelength range. Prog. Astron. Aeronaut., 82:68-80, 1982.

7 Alexandre Martin, Ioana Cozmuta, Michael J Wright, and Iain D Boyd. Kinetic rates for gas-phase chemistry of phenolic-based carbon ablator in atmospheric air. Journal of Thermophysics and Heat Transfer, 29(2):222-240, 2015.

8 K. A. Gingerich, H. C. Finkbeiner, and R. W. Schmude Jr. Enthalpies of formation of small linear carbon clusters. Journal of the American Chemical Society, 116(9):3884-3888, 1994.

9 K. S. Pitzer and E. Clementi. Large molecules in carbon vapor. Journal of the American Chemical Society, 81(17):4477-4485, 1959.

10 E. Clementi. Electronic states in the $\mathrm{C}_{4}$ molecule. Journal of the American Chemical Society, 83(22):45014505, 1961.

11 A. Van Orden and R. J. Saykally. Small carbon clusters: spectroscopy, structure, and energetics. Chemical Reviews, 98(6):2313-2358, 1998.

12 A. Karton, A. Tarnopolsky, and J. M. L. Martin. Atomization energies of the carbon clusters $\mathrm{Cn}(\mathrm{n}=2-10)$ revisited by means of W4 theory as well as density functional, Gn, and CBS methods. Molecular Physics, 107(8-12):977-990, 2009.

13 L. A. Curtiss, K. Raghavachari, G. W. Trucks, and J. A. Pople. Gaussian-2 theory for molecular energies of first-and second-row compounds. The Journal of Chemical Physics, 94(11):7221-7230, 1991.

14 L. A. Curtiss, K. Raghavachari, P. C. Redfern, V. Rassolov, and J. A. Pople. Gaussian-3 (G3) theory for molecules containing first and second-row atoms. The Journal of Chemical Physics, 109(18):7764-7776, 1998.

15 L. A. Curtiss, P. C. Redfern, and K. Raghavachari. Gaussian-4 theory. The Journal of Chemical Physics, 126 (8):084108, 2007.

16 Jan ML Martin and Glênisson de Oliveira. Towards standard methods for benchmark quality ab initio thermochemistryw1 and w2 theory. The Journal of chemical physics, 111(5):1843-1856, 1999.

17 A. D. Becke. Density-functional thermochemistry. III. the role of exact exchange. The Journal of Chemical Physics, 98:5648-5652, 1993. 
18 A. D. Becke. Beckes three parameter hybrid method using the LYP correlation functional. The Journal of Chemical Physics, 98:5648-5652, 1993.

19 T. H. Dunning Jr. Gaussian basis sets for use in correlated molecular calculations. I. the atoms boron through neon and hydrogen. The Journal of Chemical Physics, 90(2):1007-1023, 1989.

20 R. A. Kendall, T. H. Dunning Jr, and R. J. Harrison. Electron affinities of the first-row atoms revisited. systematic basis sets and wave functions. The Journal of Chemical Physics, 96(9):6796-6806, 1992.

${ }^{21}$ M. J. Frisch, G. W. Trucks, H. B. Schlegel, G. E. Scuseria, M. A. Robb, J. R. Cheeseman, G. Scalmani, V. Barone, B. Mennucci, G. A. Petersson, et al. Official gaussian 09 literature citation. Availabl e: www. gaussian. com/g_tech/g_ur/m_citation. htm. Accessed, 9, 2014.

22 John F Stanton and Rodney J Bartlett. The equation of motion coupled-cluster method. a systematic biorthogonal approach to molecular excitation energies, transition probabilities, and excited state properties. The Journal of chemical physics, 98(9):7029-7039, 1993.

23 B. Ruscic, R. E. Pinzon, M. L. Morton, G. von Laszevski, S. J. Bittner, Sandeep G Nijsure, Kaizar A Amin, M. Minkoff, and A. F. Wagner. Introduction to active thermochemical tables: Several key enthalpies of formation revisited. The Journal of Physical Chemistry A, 108(45):9979-9997, 2004.

24 B. Ruscic, R. E. Pinzon, G. von Laszewski, D. Kodeboyina, A. Burcat, D. Leahy, D. Montoy, and A. F. Wagner. Active thermochemical tables: thermochemistry for the 21st century. In Journal of Physics: Conference Series, volume 16, page 561. IOP Publishing, 2005.

25 B. Ruscic. Active thermochemical tables (atct) values based on ver. 1.118 of the thermochemical network, 2015. available at ATcT. anl. gov, 2016.

26 Daniel Richard Stull and Harold Prophet. Janaf thermochemical tables. Technical report, National Standard Reference Data System, 1971.

27 Lev Veniaminovich Gurvich and I Veyts. Thermodynamic Properties of Individual Substances: Elements and Compounds, volume 2. CRC press, 1990.

28 D. W. Schwenke. Unpublished.

29 H. Werner, P. J. Knowles, G. Knizia, F. R. Manby, and M. Schütz. Molpro: A general-purpose quantum chemistry program package. Wiley Interdisciplinary Reviews: Computational Molecular Science, 2(2):242253, 2012.

30 Bonnie J McBride, Sanford Gordon, and Martin A Reno. Coefficients for calculating thermodynamic and transport properties of individual species. 1993. 